\title{
Cryptorchidie de l'enfant: syndrome ou symptôme?
}

\author{
C. SultaN*, ** S. Lumbroso** \\ *Consultation d'Endocrinologie Pédiatrique, Service de Pédiatrie I, \\ Hôpital Arnaud de Villeneuve, 34295 Montpellier ; \\ **Unité BEDR, Centre de Recherche INSERM, Hôpital Lapeyronie, 34295 Montpellier
}

\section{RESUME}

La cryptorchidie de l'enfant est une affection fréquente dont la prise en charge thérapeutique reste discutée. Le diagnostic clinique doit s'assurer du caractère permanent de la cryptorchidie et rechercher l'existence de manifestations associées.

L'exploration endocrinienne devra comporter un dosage de la testostérone plasmatique et un test de stimulation par les HCG. Trois types d'anomalies sont responsables du développement de la cryptorchidie : anomalies anatomiques, histologiques et hormonales.

Le traitement médical, aussi précoce que possible sera, en cas d'échec, suivi d'une orchidopexie chirurgicale.

Le pronostic est dominé par les risques de stérilité et de cancer du testicule.

Mots clés : cryptorchidie, diagnostic clinique, traitement, revue générale.

La cryptorchidie correspond à un arrêt de la migration testiculaire dans le scrotum. C'est la plus fréquente des anomalies de la différenciation sexuelle (prises au sens large du terme), dont la prise en charge reste difficile et dont le pronostic est dominé par les risques de stérilité et de cancer du testicule.

\section{DIAGNOSTIC DE LA CRYPTORCHIDIE}

\section{Définition}

Tout testicule non descendu ou incomplètement descendu est cryptorchide : ce testicule se trouve donc en permanence en dehors du scrotum, soit intra-abdominal, soit dans le canal inguinal, son abaissement manuel est impossible ou suivi d'une réascension dès que cesse la traction.

Cette définition exclut les testicules rétractiles et les testicules ectopiques:

- un testicule rétractile (ou oscillant, ou ascenseur) est capable de descendre spontanément dans le scrotum,

- un testicule ectopique se trouve à l'extérieur du trajet normal de la migration : région inguinale superficielle, périnéale voire crurale ou fémorale !

\section{Fréquence}

La fréquence de la cryptorchidie est de l'ordre de 0,8 à $1,6 \%$ chez les garçons (3-4 $\%$ des nouveau-nés à terme, $20 \%$ nouveaunés prématurés) : la migration testiculaire qui s'effectue principalement au cours du 3 ème trimestre de gestation peut donc s'achever pendant les premiers mois de la vie, mais certainement pas au-delà.

\section{Diagnostic clinique}

Le diagnostic positif repose essentiellement sur l'examen clinique réalisé soit lors d'un 
examen systématique, soit plus rarement à l'occasion d'une complication, soit enfin à la demande de la mère.

L'examen clinique doit être attentif, prolongé, l'enfant est placé en décubitus dorsal ou en tailleur, complètement relaxé et mis en confiance.

Cet examen a pour but de préciser la situation du testicule (Figure 1) s'il est palpable, son volume, sa consistance, son caractère abaissable ou non par traction manuelle. Si la cryptorchidie est unilatérale, le volume et la consistance du testicule controlatéral doivent être précisés. $\mathrm{Si}$ aucun testicule n'est perçu il faut alors évoquer une cryptorchidie bilatérale (20 $\%)$ ou exceptionnellement une anorchidie congénitale $(<1 \%)$.

L'examen doit enfin rechercher, systématiquement, des associations pathologiques :

- de la verge (micropénis, hypospadias)

- des syndromes dysmorphiques,

- des retards mentaux.

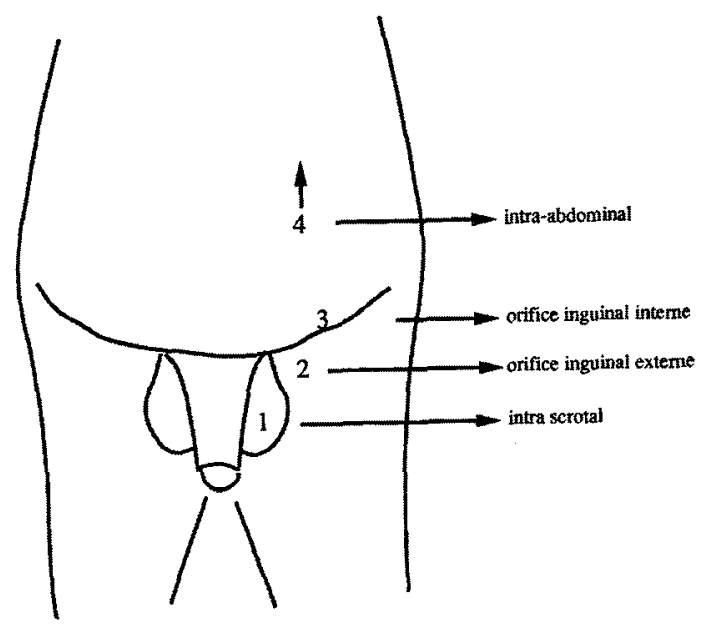

Figure 1 : les différentes positions du testicule cryptorchide.

\section{Explorations endocriniennes}

L'exploration hormonale qui repose sur l'étude de la testostérone plasmatique basale, de la réserve hypophysaire en LH-FSH sous LH-RH et sur la réponse de la testostérone plasmatique après 7 injections de 1500 unités de HCG, doit être entreprise à bon escient. Lorsque l'on craint une anorchidie bilatérale, le test à l'HCG nous semble indispensable. Si l'on suspecte une insuffisance gonadotrope, le test au LH-RH peut s'avérer utile [10].

Dans la cryptorchidie commune, la sécrétion de testostérone est inférieure à la normale en période néonatale, à la limite inférieure de la normale jusqu'au début de la puberté. De la même façon, la réponse des cellules de Leydig à la stimulation reste significativement inférieure à la normale.

Le dosage de la LH plasmatique de base, comme l'étude de la réserve en LH serait (pour certains) en faveur d'un déficit partiel, précoce et transitoire de la sécrétion hypophysaire de LH [5].

L'élévation de la FSH plasmatique en période pubertaire est parallèle :

- à la réduction du nombre de spermatogonies,

- à l'importance des lésions tubulaires ;

elle est responsable de l'augmentation compensatrice du testicule controlatéral.

Lorsqu'elle a été mesurée, l'inhibine est normale ou discrètement réduite, par contre l'hormone anti-mullerienne est basse, en relation avec l'atteinte sertolienne.

$\mathrm{Au}$ total, dans notre expérience, la réponse biologique des cellules de Leydig à la stimulation par les HCG, assortie d'un examen clinique minutieux de la migration testiculaire, nous paraissent indispensables, en première intention, devant une cryptorchidie de l'enfant. 
Au-delà, et en fonction de l'orientation diagnostique, un test au LH-RH, un caryotype sont parfois nécessaires.

\section{Explorations échographiques}

Dans certains cas, l'échographie testiculaire va permettre de confirmer la présence de testicules en précisant leur situation anatomique. Cet examen n'est cependant pas réalisé en routine.

\section{DIAGNOSTIC DIFFERENTIEL}

Par définition, nous avons éliminé le testicule rétractile, en général unilatéral qui représente l'une des erreurs les plus courantes. Il faut toutefois surveiller ces enfants pour vérifier qu'en période pubertaire le testicule s'est bien fixé dans la bourse.

L'anorchidie congénitale bilatérale se différencie facilement de la cryptorchidie par les données de l'exploration endocrinienne : des valeurs de FSH, LH plasmatiques élevées, une testostérone plasmatique effondrée qui ne répond pas au test de stimulation par les HCG, signent ce diagnostic.

La monorchidie est, par contre, plus difficile à identifier : la réponse hormonale aux explorations endocriniennes justifie, devant un testicule unique hypertrophique, la pratique d'une échographie voire d'une tomodensitométrie. L'imagerie par résonnance magnétique serait plus performante. La laparoscopie est l'investigation de choix en cas de testicule(s) non palpable(s), lorsque l'épreuve de stimulation par les HCG est négative.

Les pseudo-hermaphrodismes féminins : toute cryptorchidie bilatérale doit faire écarter systématiquement un pseudohermaphrodisme féminin avec virilisation complète des organes génitaux externes (type V de Prader). Le diagnostic est affirmé par l'absence de gène SRY, l'élévation importante de la production de $17-\mathrm{OH}$ progestérone, d'androgènes qui signent le bloc en 21-OH responsable d'une hyperplasie congénitale de la surrénale

\section{ETIOPATHOGENIE}

De nombreux facteurs participent à la migration testiculaire normale :

- anatomiques,

- histologiques,

- hormonaux.

Il est donc légitime d'évoquer leur participation au développement de la cryptorchidie.

\section{Anomalies anatomiques}

Les anomalies anatomiques associées à la cryptorchidie sont fréquentes et font évoquer l'hypothèse d'une affection loco-régionale :

- la persistance du canal péritonéo-vaginal avec hernie inguinale est fréquente (30 à $90 \%$ des cas) ;

- une terminaison anormale, du gubernaculum testis a été souvent retrouvée : insertion en dehors du scrotum, étalement en éventail de ce ligament ;

- anomalies de l'épididyme (3-5\%) : non accollement au testicule (partiel ou total) et risque d'absence de continuité de la voie afférente des spermatozoïdes ;

- anomalies des déférents (7-9\%) : allongement anormal, non coalescence avec le testis, atrésie , absence de ces canaux ;

- anomalies des vaisseaux spermatiques: une artère spermatique anormalement courte rendra souvent difficile la descente testiculaire chirurgicale.

\section{Anomalies histologiques}

Les altérations structurales du testicule cryptorchide ont fait l'objet d'études extensives dont les résultats ont conduit à une prise en charge médicale ou chirurgicale plus précoce et plus énergique. 
Deux données importantes émergent des travaux réalisés chez l'enfant cryptorchide, à partir de biopsies testiculaires :

- l'atteinte précoce des tubes séminifères [2],

- la relative fréquence d'une altération du testicule controlatéral, en place [7].

La réduction importante du nombre des spermatogonies (et de leur diamètre) visible en MO comme en ME constitue l'élèment le plus préoccupant du pronostic de la cryptorchidie (Figure 2). Comme l'a précisé Hadiziselimovic [2] cette altération apparaît très précocément, dès la 3 ème année de vie (Figure 2) et constitue un argument essentiel pour le timing précoce de l'orchidopexie. Cet effondrement de la concentration en spermatogonies [11] est associé à une réduction du volume tubulaire, à un certain degré de fibrose interstitielle ; fréquemment, on observe également une diminution du nombre de cellules de Leydig.

Le testicule intra-scrotal controlatéral présente, avec une certaine fréquence, des altérations de la lignée germinale qui ont fait évoquer l'hypothèse d'une anomalie primitive (maladie testiculaire) à l'origine de la cryptorchidie (Figure 3).

\section{Anomalies endocriniennes}

De nombreux arguments démontrent le rôle clé de la testostérone dans la descente testiculaire : en effet toute altération de l'un des éléments de l'axe gonadotrope est génératrice de cryptorchidie (Tableau 1).

- déficit en LH-RH,

- défaut de sécrétion en $\mathrm{LH}$,

- défaut de synthèse de la testostérone,

- déficit en $5 \alpha$-Réductase,

- résistance aux androgènes.

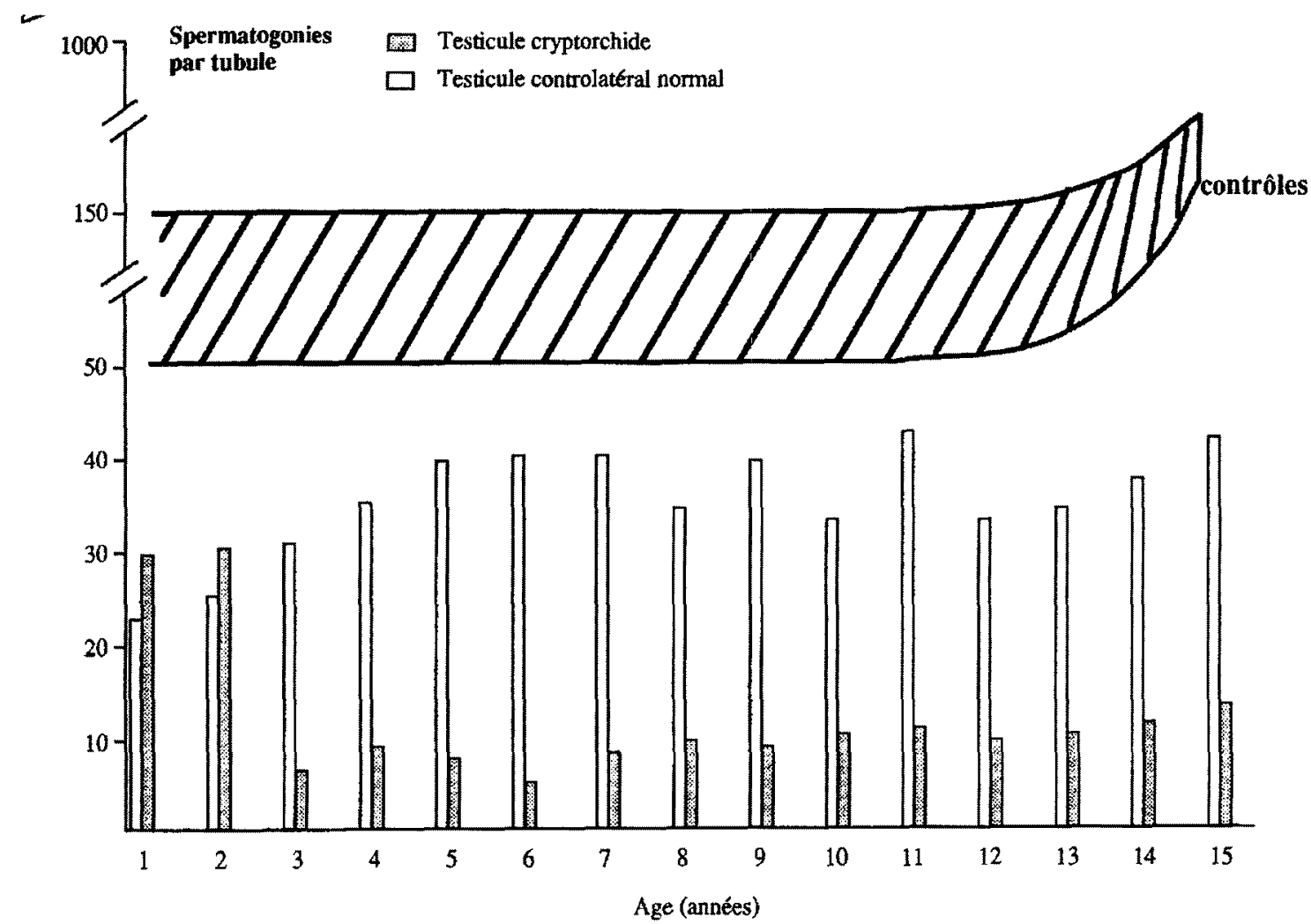

Figure 2 : Comparaison de la concentration en spermatogonies par tubule, chez les enfants porteurs d'une cryptorchidie unilatérale. 

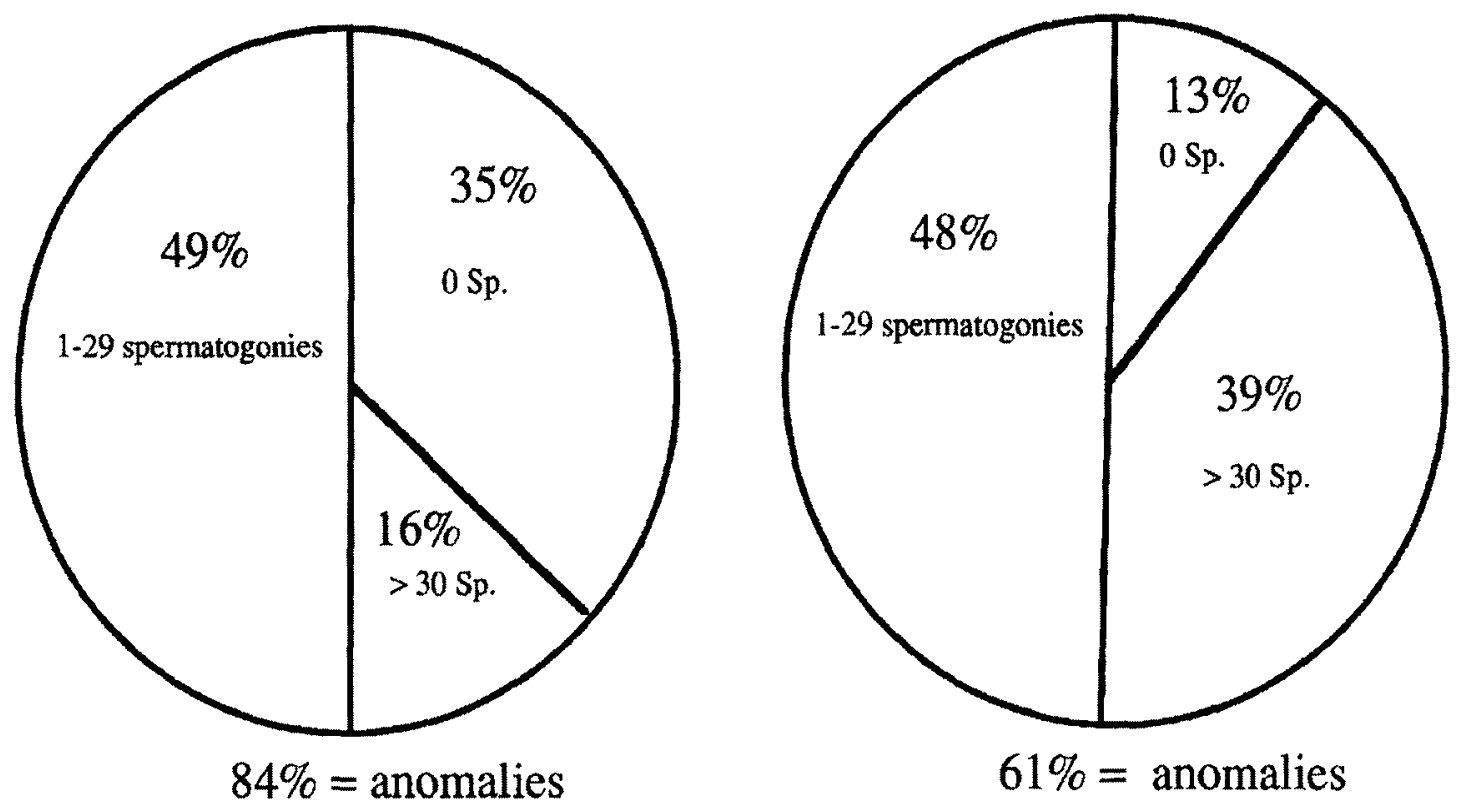

Figure 3 : Analyse de la concentration en spermatogonies de biopsie testiculaire d'enfants présentant une cryptorchidie unilatérale.

Tableau 1 : Principales causes endocriniennes des cryptorchidies.

\begin{tabular}{ll}
\hline Axe gonadotrope & Anomalies cliniques \\
\hline hypothalamus & $\begin{array}{l}\text { - syndrome de Kallmann } \\
\text { - syndrome de Willy Prader } \\
\text { - anencéphalie }\end{array}$ \\
hypophyse & - aplasie hypophysaire \\
testicule & - persistance des canaux de Müller \\
& - testostérone
\end{tabular}


Non seulement la sécrétion, mais aussi l'action normale des androgènes au niveau cellulaire ( $5 \alpha$-Réductase et récepteur) est également impliquée dans la migration testiculaire [4].

Par contre, comme nous l'avons vu, l'exploration hormonale de la cryptorchidie commune n'est pas univoque et le concept de déficit en LH largement controversé.

\section{DIAGNOSTIC ETIOLOGIQUE}

Plusieurs monographies ont mis en lumière la diversité étiologique des cryptorchidies de l'enfant : on peut schématiquement l'aborder sous deux rubriques :

- Cryptorchidie = syndrome,

- Cryptorchidie = symptôme.

En faveur de la cryptorchidie commune (Tableau 2), véritable syndrome à elle seule, plaident :

- sa fréquence : 80 - $90 \%$ des cryptorchidies de l'enfant,

- son association à des malformations anatomiques génitales,

- son caractère génétique : l'incidence de la cryptorchidie chez le père, ou chez un frère, est de 5 à $6 \%$. Il existe de plus un

\section{Tableau $2:$ Cryptorchidie $=$ symdrome $?$}

\section{1. facteurs génétiques}

- ATCD de cryptorchidie chez le père $6 \%$ le frère $4 \%$

- liaison génétique : HLA A-11, A-23

- anticorps anti-LH

\section{2. facteurs endocriniens}

- déficit partiel en LH

- déficit lutéal chez la mère

\section{3. anomalies anatomiques associées}

- hernies inguinales : 30 à $89 \%$

- anomalie de la tête de l'épididyme : 3 à $5 \%$

- anomalie du déférent : 7 à $9 \%$ déséquilibre de liaison du groupe HLAA11 et HLA A23 lors de cryptorchidies [6].

Dans 10 à $20 \%$ des cas, la cryptorchidie est un symptôme révélateur d'une affection à rechercher (Tableau 3) :

- anomalie de l'axe gonadotrope,

- anomalie de la différenciation sexuelle,

- syndrome polymalformatif : la cryptorchidie n'est qu'un élèment constitutif du tableau clinique [9] correspondant à différents syndromes plus ou moins bien individualisés,

- anomalie chromosomique.

\section{TRAITEMENT}

Le pronostic des cryptorchidies est dominé par le risque de stérilité et celui de tumeur testiculaire. La prise en charge thérapeutique, qu'elle soit médicale ou chirurgicale, a donc pour objectifs essentiels la réduction sinon la prévention de ces deux risques.

\section{Le traitement médical : hormonal}

Il paraît légitime d'y recourir en première intention : si les résultats rapportés dans la littérature sont discordants, cela tient sans

\section{Tableau 3 : Cryptorchidie : symptôme?}

1. affection endocrinienne - anomalie de l'axe gonadotrope

- ambiguïté sexuelle

\section{2. anomalie chromosomique}

- syndrome de Klinefelter

- syndrome de Noonan

- trisomie 21

- syndrome de Fanconi

\section{3. ensemble polymalformatif} - syndrome de Cornelia De Lange

- syndrome de Silver-Russel

- syndrome d'Aarskog

- anomalies du tube neural 
doute au nombre et au rythme des injections d'HCG qui différent d'un groupe à l'autre. Pour notre part, la prescription de 7 injections d'HCG (1500 UI) un jour sur deux nous semble indiquée quelle que soit la position anatomique du testicule : dans 20 à 30 $\%$ des cas la descente complète du testicule est obtenue. Toute descente partielle ou à fortiori, toute absence de migration testiculaire justifie un traitement chirurgical.

\section{Le traitement chirurgical : l'orchido- pexie}

Il est habituellement envisagé après échec du traitement hormonal. Il s'agit d'une intervention minutieuse surtout chez l'enfant très jeune.

L'orchidopexie comprend la libération du testicule enveloppé de sa vaginale et du cordon. Elle comporte également la suppression d'une communication péritonéo-vaginale si elle existe, la séparation du déférent des vaisseaux spermatiques, la suppression des éléments fibreux. La dissection du pédicule vasculaire apparaît, pour les chirurgiens, comme un moment essentiel de lintervention chirurgicale.

\section{Les choix}

Les altérations histologiques observées dès la troisième année de vie justifient un traitement précoce, médical et/ou chirurgical entre 1 et 2 ans.

Le traitement médical ne fera appel qu'aux HCG, l'utilisation de la LHRH par voie nasale n'a pas fait la preuve de son efficacité $[3,8]$.

\section{CONCLUSION}

La cryptorchidie commune est certainement un syndrome qu'il faut prendre en charge précocément. Dans d'autres circonstances, la cryptorchidie peut révéler une pathologie endocrinienne ou un syndrome polymalformatif complexe. Un traitement spécifique devra être alors proposé.
Si de nombreuses incertitudes persistent encore à l'heure actuelle,sur l'étiopathogénie, sur la meilleure approche thérapeutique, une coopération entre pédiatres et urologues est indispensable non seulement pour adapter la décision à chaque patient, mais envisager une étude longitudinale (jamais réalisée à ce jour) des conséquences à long terme de la cryptorchidie, de son expression, de son traitement, sur la lignée germinale

\section{REFERENCES}

1. Berkowitz G.S., R.H. LaPinski, S.E. DOlgin, J.G. Gazella, C.A. Bodian, I.R. Holzman : Prevalence and natural history of cryptorchidism- Pediatrics $1993 ; 92: 44-9$.

2. Hadiziselimovic F. : Pathogenesis and treatment of undescended testes - European Journal of Pediatrics $1982 ; 139: 255-265$.

3. HAdiZiselimovic F. : Treatment of cryptochidism with GnRH- Urol Clini North Am $1988 ; 9$ : 413420 .

4. Hutson J.M, Donohue P.K : The hormonal control of testicular descent - Endocrin Rev 1986; $7: 279$ 283.

5. Јов J.C, Pierson M : Endocrinologie pédiatirque et croissance- Flammarion Medecine Sciences 1978 .

6. Martinetti M, Maghnie M. Salvaneschi L et Al. : Immunogenetic and hormonal study of cryptochidism.- J. Clin Endocrinol Metab $1992 ; 74$ : 39-42.

7. MENGEL W, KECKER WCH, MoRITZ : The treatment of the maldescended testis under special consideration of the moment of surgical treatment - Bierich JR, Rager K, Ranke HB (eds) Maldescensus testis. 1977 Urban et Schwarzenberg, Munchen.

8. Raffer J, Handelsman D.J, Swerdloff R.S et AL. : Hormonal therapy of cryptorchidism- N. Engl. J. Med 1986 ; $314: 466-70$.

9. RAPPAPORT $R$ : Cryptochidism in relation to various disorders. Pediatr. Adolesc. Endocr. 1979 ; 6 : 154159.

10. SAENGER P, O.E.REITER : Management of cryptochjdism- TEM 1992 ; Vol 3, No 7 249-253.

11. Saito S, Kumamoto Y : The number of spermatogonia in various congenital testicular disorders- J. Urol 141: 1166-1168. 


\section{ABSTRACT}

Cryptorchidism : symptom or syndrom ?

Ch. Sultan, S. Lumbroso

Cryptorchidism is a frequent disease of children whose management remains controversial. Clinical diagnosis of cryptorchidism must assess the permanent location of the testis outside scrotum and must look for associated malformations. Endocrine investigation should include an evaluation of basal plasma testosterone and after an HCG stimulation test. Development of cryptorchidism is due to anatomical, histological and endocrine abnormalities.

Medical treatment should be realised as early as possible. If testis descent does not occur, orchidopexy must be performed. Long-term effects of cryptorchidism are defined by infertility and testis cancer.

Key-Words : cryptorchidism, clinical evaluation, treatment, general review. 